\title{
Os impactos do Chemsex na saúde pública mundial: um estudo sobre uma perigosa prática sexual entre homens
}

\section{RESUMO}

O objetivo deste artigo foi de descrever o perfil de publicações sobre Chemsex expressos na literatura internacional e seus impactos na saúde pública. Método: revisão integrativa de literatura realizada nos bancos de dados dos periódicos CAPES, Biblioteca Virtual de Saúde (BVS) e PubMed, utilizando-se a expressão Chemsex. Resultados: Foram analisados 146 artigos publicados em língua inglesa em diferentes países, eles foram agrupados em 5 categorias, a saber: Principais substâncias psicoativas; Infecções sexualmente transmissiveis/Morbidades; Comportamentos de risco; Aspectos epidemiológicos; e Abordagens terapêuticas. Essa prática tem aumento a vulnerabilidade dos indivíduos, intensificada por "estados de faltas", associando-se também à distúrbios emocionais, acarretando a busca desordenada pelo prazer, associada ao consumo de múltiplas drogas.

DESCRITORES: Usuários de Drogas; Infecções por HIV; Grupos de Risco.

\section{ABSTRACT}

The purpose of this paper was to describe the profile of publications on Chemsex expressed in the international literature and their impacts on public health. Method: integrative literature review conducted in the databases of CAPES journals, Virtual Health Library (VHL) and PubMed, using the expression Chemsex. Results: We analyzed 146 articles published in English in different countries, they were grouped into 5 categories, namely: Main psychoactive substances; Sexually Transmitted Infections/ Morbidities; Risk behaviors; Epidemiological aspects; and Therapeutic Approaches. This practice has increased the vulnerability of individuals, intensified by "fault states", also associated with emotional disturbances, leading to the disordered pursuit of pleasure associated with multiple drug use. KEYWORDS: Drug Users; HIV Infections; Risk Groups.

\section{RESUMEN}

El propósito de este documento fue describir el perfil de publicaciones sobre Chemsex expresadas en la literatura internacional y sus impactos en la salud pública. Método: revisión bibliográfica integradora realizada en las bases de datos de revistas CAPES, Biblioteca Virtual en Salud (BVS) y PubMed, utilizando la expresión Chemsex. Resultados: Analizamos 146 artículos publicados en inglés en diferentes países, se agruparon en 5 categorías, a saber: principales sustancias psicoactivas; Infecciones/ Morbilidades de Transmisión Sexual; Conductas de riesgo; Aspectos epidemiológicos; y enfoques terapéuticos. Esta práctica há aumentado la vulnerabilidad de las personas, intensificada por "estados de falla", también asociados con trastornos emocionales, lo que lleva a la búsqueda desordenada del placer asociada con el uso múltiple de drogas.

PALABRAS CLAVE: Consumidores de Drogas; Infecciones por VIH; Grupos de Riesgo.

RECEBIDO EM: 21/10/2019 APROVADO EM: 21/10/2019

\section{Roni Robson da Silva}

Acadêmico de enfermagem da Universidade Veiga de Almeida (UVA)

\section{Leandro Andrade da Silva}

Enfermeiro. Pós-Doutor pelo Programa de Pós-graduação em Enfermagem da Universidade do Estado do Rio de Janeiro (PPGEnf/UERJ). Especialista em Saúde Mental e Atenção Psicossocial pela Escola Nacional de Saúde Pública Sérgio Arouca/ENSP da Fundação Oswaldo Cruz (FIOCRUZ). Professor do Curso de Graduação em Enfermagem da Universidade Veiga de Almeida (UVA). 


\section{Maria Virginia Godoy da Silva}

Enfermeira. Especia+lista em Enfermagem em Centro Cirúrgico e Central de Material e Esterilização e Recuperação Anestésica pela Escola de Enfermagem da Universidade de São Paulo (EEUSP). Doutora em Enfermagem pela EEUSP. Professora Adjunto da Enfermagem da Faculdade de Enfermagem da Universidade do Estado do Rio de Janeiro (FEUERJ) e Professora do Curso de Enfermagem da Universidade Veiga de Almeida (UVA).

\section{Milena Preissler das Neves}

Acadêmica de enfermagem da Universidade Veiga de Almeida (UVA).

\section{Myllena Miguel dos Santos da Silva}

Acadêmica de enfermagem da Universidade Veiga de Almeida (UVA).

\section{Márcio Tadeu Ribeiro Francisco}

Enfermeiro. Doutor em Saúde Coletiva. Livre docente do Departamento de Fundamentos de Enfermagem da Universidade do Estado do Rio de Janeiro. Coordenador Geral do Curso de Graduação em Enfermagem da Universidade Veiga de Almeida. Pesquisador Líder do Grupo de pesquisa Laboratório de Abordagens Culturais e Cuidados em Saúde - LACES da Universidade Veiga de Almeida.

\section{Cristiano Bertolossi Marta}

Enfermeiro. Pós-Doutor em Enfermagem. Professor Adjunto do Departamento de Fundamentos de Enfermagem da Faculdade de Enfermagem da Universidade do Estado do Rio de Janeiro. Coordenador Geral da Pós-Graduação de Enfermagem em Alta Complexidade da Universidade Veiga de Almeida. Diretor de Pesquisa da Universidade Veiga de Almeida. Pesquisador Líder do Núcleo de Avaliação de Tecnologias e Economia em Saúde e Segurança do Paciente (NATESSP) da Universidade Veiga de Almeida.

\section{INTRODUÇÃO}

$\mathbf{R}$ egistos do uso de substâncias psicoativas remontam à pré-história; há mais de dois mil anos, no templo de Elêusis, na antiga Grécia, era o centro espiritual e místico mais importante da cultura helenística. Eram celebrados os mistérios dedicados os três deuses ctônicos - Deméter e o ciclo da vida e da morte; Dionísio e a transgressão dos prazeres do corpo; e Orfeu e o desejo de purificação e ascese da alma. A utilização de substâncias que alteram estados de consciência é uma prática comum entre humanos ${ }^{(1)}$.

De acordo com o Boletim Epidemiológico publicado pela Organização Mundial de Saúde (OMS) em junho de 2019, ocorreram melhorias nos dados sobre a aquisição do vírus HIV. Através das Profilaxia Pós-Exposição (PEP) ao HIV, compreendida como uma medida de prevenção de urgência à infecção pelo HIV, hepatites virais e outras infecções sexualmente transmissíveis (IST), que consiste em aplicar medicamentos antirretrovirais que reduzem o risco de infecção em situações de exposição ao vírus. A PEP é uma tecnologia utilizada na chamada prevenção combinada, cujo principal objetivo é ampliar as formas de intervenção para atender às ne- cessidades e possibilidades de cada pessoa e evitar novas infecções pelo HIV, hepatites virais e outras IST. Geralmente indicada em situações que haja risco de contágio em função de situações de violência sexual; relação sexual desprotegida (sem o uso de preservativo ou com rompimento do mesmo) e acidente ocupacional - com instrumentos perfurocortantes ou contato direto com material biológico ${ }^{(2)}$.

Outra estratégia utilizada é a Profilaxia Pré-Exposição (PrEP) ao HIV, este novo método adotado no Brasil, já existente em diferentes países, consiste na tomada diária de um comprimido que impede que o vírus causador da Aids infecte o organismo, antes de a pessoa ter contato com $\mathrm{o}$ vírus. A PrEP não protege o indivíduo de outras ISTs, tais como sífilis, clamídia e gonorréia e, portanto, deve ser combinada com outras formas de prevenção, como a utilização de preservativos. Lamentavelmente, alguns indivíduos banalizam a prevenção combinada e mantêm relações sexuais de forma desprotegida ${ }^{(2)}$.

Os comportamentos de risco à saúde geram grande impacto na saúde pública mundial, dentre eles destacam-se: o consumo em demasia de drogas legais e ilegais, envolvimento em situações de violência e condutas sexuais desprotegidas. Na busca por pra- zer, indivíduos com dificuldades de manter relacionamentos afetivos duradouros utilizam de substâncias entorpecentes como uma espécie de catalisador para mascarar sentimentos negativos. Focando apenas no ato sexual, distanciam-se da afetividade, do carinho, do amor ou sentimentos mútuos de bem querer, deixando o gostar de lado e limitando, erroneamente, sexualidade ao sexo. Na busca por aumento do prazer e da potência sexual através de substâncias, aumentam-se as vulnerabilidades emocional e física, gerando o abandono e a rejeição ${ }^{(3)}$.

O termo Chemsex é utilizado para descrever as práticas sexuais potencializadas pelo consumo de drogas e de várias substâncias químicas. É uma prática sexual que pode provocar sérios danos físicos e emocionais. Esta modalidade sexual leva as pessoas a fazer sexo nonstop sob o efeito de drogas, os efeitos podem durar longas horas e até mesmo por dias, associados incialmente ao sexo em grupo entre homens. No entanto, existem registros desta prática que se generalizou. O impacto desta modalidade é um sério problema de saúde pública, haja vista a possibilidade para além da dependência química, é a aquisição de ISTs e os efeitos negativos das drogas podem ainda dar origem a problemas mentais associados à 
ansiedade, psicoses e, ainda, tendências suicidas ou ataques de pânico ${ }^{(4)}$.

A inexistência de estudos significativos publicados no Brasil não significa que esta prática sexual não ocorra no país, faz-se necessária a realização de estudos, com base em aplicativos de encontros; redes sociais, festas etc. A fim de localizar os adeptos da prática do Chemsex. Esta prática tem aumentado a vulnerabilidade dos indivíduos, intensificada por "estados de faltas", associando-se também à distúrbios emocionais, acarretando a busca desordenada pelo prazer, associada ao consumo de múltiplas drogas ${ }^{(5)}$.

O estudo tem como questão de pesquisa: O que a literatura científica vem produzindo sobre Chemsex e o impacto desta prática sexual na saúde pública? Sendo assim, este artigo objetivou descrever o perfil de publicações sobre Chemsex expressos na literatura internacional e seus impactos na saúde pública.

\section{METODOLOGIA}

Escolheu-se a Revisão Integrativa de
Literatura (RIL), cuja busca por estudos científicos considera critérios de inclusão e exclusão explícitos, avaliação da qualidade metodológica e na quantificação das publicações aplica-se técnicas estatísticas. Foram respeitadas rigorosamente as seis etapas da RIL.

Primeira - Escolha da questão de pesquisa: $\mathrm{O}$ que a literatura científica vem produzindo sobre Chemsex e o impacto desta prática sexual na saúde pública? Segunda - Busca nas bases de dados eletrônicas Medline, LILACS e BDENF, via portais PubMed e a Biblioteca Virtual de Saúde (BVS) e através do Portal de Periódicos Capes. Realizada em setembro de 2019, utilizando a expressão Chemsex, pois inexistem descritores padronizados nos Descritores em Ciências da Saúde (DECs) que congreguem termos aproximados aos de interesse desta RIL.

Terceira - Seleção dos trabalhos segundo os critérios de inclusão: publicações de estudos sociopoéticos do período de 2015 a 2019; idioma português, inglês e espanhol; com resumos e textos

\section{Figura 1. Síntese dos resultados da revisão sistemática. Rio de Janeiro, RJ,} Brasil, 2019.

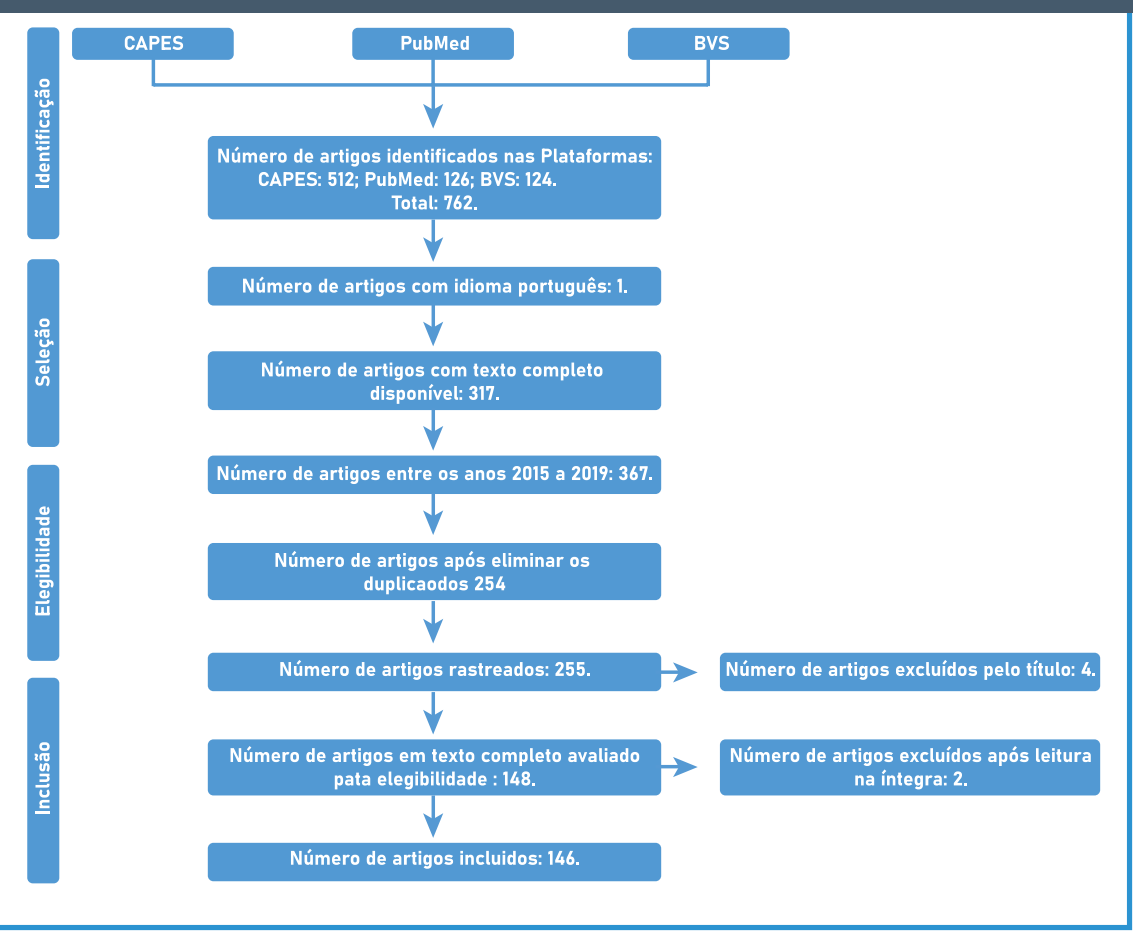

completos disponíveis online nas bases de dados citadas. Critério de exclusão, artigos cuja descrição não se referia à temática do estudo. Identificou-se 762 trabalhos, dos quais 146 foram selecionados para esta RIL, apresentados através do fluxograma PRISMA ${ }^{(6)}$. Quarta - Garantia do rigor metodológico, elaborando-se formulário composto por variáveis relacionadas à identificação do artigo: ano e periódico de publicação; caracterização dos estudos; titulação dos autores; sujeitos de pesquisa e síntese dos resultados. Aos dados produzidos, aplicou-se a estatística descritiva simples e análise categorial de Bardin, sendo estes representados graficamente em quadros demonstrativos.

Quinta - Análise crítica dos trabalhos selecionados, comparando-se os conhecimentos teóricos, a identificação de conclusões e implicações resultantes da RIL. $\mathrm{O}$ que possibilitou a compreensão sobre o estado da arte da produção de conhecimento na área de saúde publica sobre o impacto do Chemsex.

Sexta e última etapa - Revisão/síntese do conhecimento produzido incluindo-se as informações que permitiram avaliar a pertinência dos princípios e pressupostos do método utilizado. Nesta RIL, a identificação do nível de evidência dos artigos considerou as de acordo com Agency for Healthcare Research and Quality (AHRQ). Destaca-se que, considerando os dados produzidos nesta RIL, delimitou-se 5 categorias/áreas temáticas.

Uma limitação do estudo foi a baixa produção de artigos em português e a inexistência de pesquisas realizadas no Brasil sobre essa temática. Quase em sua totalidade, os artigos publicados foram em língua inglesa, acessados via online.

Os resultados foram esquematizados através do fluxograma PRISMA, expressos na Figura 1. Na busca inicial, foram identificados 762 artigos, dos quais após aplicação dos critérios de exclusão permaneceram 146 publicações. Destaca-se a predominância de artigos publicados em inglês, haja vista, só existir um estudo desenvolvido sobre a temática no Brasil. 


\section{artigo}

Silva, R.R.; Silva, L.A.; Silva, M.V.G.; Neves, M.P.; Silva, M.M.S.; Francisco, M.T.R.; Marta, C.B.;

Os impactos do Chemsex na saúde pública mundial: um estudo sobre uma perigosa prática sexual entre homens

\section{RESULTADOS}

Os estudos foram apresentados no Quadro 1, agrupados em 5 categorias, a saber: Principais substâncias psicoativas; Infecções sexualmente transmissíveis/ Morbidades; Comportamentos de risco; Aspectos epidemiológicos; e Abordagens terapêuticas.

\section{DISCUSSÃO}

Os achados expressos nos estudos evidenciaram o grave impacto do Chemsex na saúde pública mundial, haja vista, esta prática ser comum em diversos continentes. Predominantemente realizada por pessoas com recursos financeiros, bom nível sociocultural e com escolaridade elevada. Metaforicamente falando, o Chemsex é a ponta de um iceberg, abaixo dela existem inúmeros problemas e comportamentos.

Neste sentido, a categoria Comportamentos de risco, representada por $32 \%$ dos estudos, revelou um pouco da conduta de adeptos do Chemsex. O que levam principalmente gays e homens que fazem sexo com outros homens, denominados pela sigla $\mathrm{HSH}$, a colocarem-se em situações de vulnerabilidade à aquisição de ISTs. Os HSH não são classificados como homossexuais, eles são heterossexuais que mantêm relações sexuais ou praticam outras modalidades recreativas sexuais com outros homens, elas podem ser esporádicas ou constantes, porém, o predomínio é o sexo com desconhecidos, justamente para não colocar em risco a imagem de heterossexuais que estes têm na sociedade. Recentemente, aflorou outro acrônimo mais preciso para definir esse grupo: Straight Men who have Sex

Quadro 1. Categorização de artigos sobre ASSIST. Rio de Janeiro, RJ, Brasil, 2019.

\begin{tabular}{|c|c|c|c|c|}
\hline CATEGORIA & TEMÁTICAS ABORDADAS & N & $\mathbf{F}$ & SÍNTESE DOS ESTUDOS \\
\hline $\begin{array}{l}\text { Principais subs- } \\
\text { tâncias psicoa- } \\
\text { tivas }\end{array}$ & $\begin{array}{l}\text { Substâncias; Psicoativas; Estimulantes } \\
\text { sexuais; Antirretroviral; Metanfetamina } \\
\text { Drogas; recreativas; Drogas injetáveis; Drogas; } \\
\text { sintéticas; Multidrogas; Padrão de uso de drogas } \\
\text { ilícitas; GHB/GBL. }\end{array}$ & 46 & $32 \%$ & $\begin{array}{l}\text { Apresentou as substâncias psicoativas mais } \\
\text { utilizadas em festas promovidas para a realização } \\
\text { do Chemsex, os estados de faltas que levaram } \\
\text { os indivíduos a amortecer suas realidades e a } \\
\text { intensificarem o prazer sexual. }\end{array}$ \\
\hline $\begin{array}{l}\text { Infecções } \\
\text { sexualmente } \\
\text { transmissíveis/ } \\
\text { Morbidades }\end{array}$ & $\begin{array}{l}\text { Sífilis; Linfadenopatia Inguinal; Câncer anal; } \\
\text { Hepatite C; HIV/AIDS; Shigella }\end{array}$ & 15 & $10 \%$ & $\begin{array}{l}\text { Descreveu as principais complicações à saúde } \\
\text { e infecç̃̃es sexualmente transmissiveis, com } \\
\text { destaque para o aumento da transmissão do HIV, } \\
\text { Hepatite C e Sífilis principalmente na Europa. }\end{array}$ \\
\hline $\begin{array}{l}\text { Comportamentos } \\
\text { de risco }\end{array}$ & $\begin{array}{l}\text { Pessoas vivendo com HIV/AIDS; Transmissão } \\
\text { de ISTs; Saúde Mental; Party and Play; Tipolo- } \\
\text { gias socio-sexuais ; Associação de drogas com } \\
\text { antirretrovirais; Interações emocionais; Testagem } \\
\text { como Prevenção; ; Motivação para essa prática; } \\
\text { Comportamento de risco para ISTs; Sexo des- } \\
\text { protegido; Sexo químico contexto sociocultural; } \\
\text { Estudo de caso; Relaçães sociais normativas; } \\
\text { Experiências individuais; Aplicativos de relaciona- } \\
\text { mentos; Traumas psicológicos; Comportamento } \\
\text { sexual; Efeitos emocionais Frequência da prática; } \\
\text { Contaminação pelo HIV/AIDS; Criminalização do } \\
\text { comportamento sexual; Overdose }\end{array}$ & 46 & $32 \%$ & $\begin{array}{l}\text { Evidenciou os comportamentos de risco que leva- } \\
\text { ram os sujeitos a praticarem o Chemsex. A consci- } \\
\text { ência da transmissão através do sexo desprotegido } \\
\text { com múltiplos parceiros, principalmente através } \\
\text { do turismo sexual; da utilização de aplicativos de } \\
\text { relacionamentos e os casos de óbitos decorrentes } \\
\text { de overdoses e complicações decorrentes de ISTs. }\end{array}$ \\
\hline $\begin{array}{l}\text { Aspectos epide- } \\
\text { miológicos }\end{array}$ & $\begin{array}{l}\text { Prevenção; Novos Casos de HIV/AIDS; Migra- } \\
\text { ção de Doenças; Estudo de Revisão; Estudo de } \\
\text { Evidência; Dados epidemiológicos; Investigação } \\
\text { epidemiológica; Prevalência do sexo com drogas; } \\
\text { Óbitos decorrentes da prática; Vulnerabilidade; } \\
\text { Padrões de transmissão de ISTs; Estudos trans- } \\
\text { versais; Estudos epidemiológicos. }\end{array}$ & 24 & $16 \%$ & $\begin{array}{l}\text { Destacou os estudos epidemiológicos e o impacto } \\
\text { da prática do Chemsex na saúde pública em dife- } \\
\text { rentes países da Europa, Ásia, Oceania e América } \\
\text { do Norte. A correlação entre substâncias entorpe- } \\
\text { centes e a vulnerabilidade para aquisição de ISTs. } \\
\text { E a escassez de estratégias de prevenção. }\end{array}$ \\
\hline $\begin{array}{l}\text { Abordagens } \\
\text { terapêuticas }\end{array}$ & $\begin{array}{l}\text { Intervenção; Realização de testagem para HIV/ } \\
\text { AIDS; Terapêutica emocional; PREP X Estig- } \\
\text { ma; Reduçãa de danos Internação Hospitalar; } \\
\text { Problema de saúde pública. Intervenção clínica. } \\
\text { Aconselhamento }\end{array}$ & 15 & $10 \%$ & $\begin{array}{l}\text { Descreveu a consciência dos indivíduos que prati- } \\
\text { cam o Chemsex, a vergonha e o estigma atrelado à } \\
\text { essa prática por alguns de seus adeptos. A dificul- } \\
\text { dade parar com a prática sexual e a quase ausência } \\
\text { de acompanhamento emocional, principalmente } \\
\text { estimulados por políticas públicas. }\end{array}$ \\
\hline
\end{tabular}


with other Men (SMSM), a tradução livre seria homens heterossexuais que fazem sexo com outros homens ${ }^{(7)}$.

Para além de acrônimos, este comportamento pode ter uma relação com a orientação sexual, que é construída, socialmente, por categorias muitas vezes rígidas, classificatórias e excludentes, com implicações que afetam as identidades individual e social. Atualmente, o respeito e a aceitação da diversidade sexual é muito maior do que no passado, porém, seja por razões relacionadas a estigmas, discriminação, criminalização de suas práticas sexuais, bem como a diversidade de lugares de sociabilidade e circulação o estigma e o preconceitos são muito fortes ${ }^{(7)}$.

Algumas pesquisas, descrevem contextos e emoções, muitas vezes, mais contributivos para a exposição ao HIV do que o conhecimento acerca dos riscos. A gestão de risco é compreendida como um processamento cognitivo, nele consideram-se cálculos realizados pela pessoa para decidir sobre dada conduta, em função do conhecimento sobre o agravo e os modos de prevenção. $\mathrm{O}$ aumento na incidência do HIV e nos casos de Aids entre os gays e HSHs, certamente, teve como causas o retrocesso moralista sobre sexualidade e gênero atrelado aos baixos investimentos públicos na prevenção do HIV que caracterizaram a última década. No Brasil e no mundo, a prevenção da epidemia do HIV entre HSH permanece por quatro décadas como um grave problema de saúde pública ${ }^{(8)}$.

Destacam-se os aplicativos de relacionamentos e demais redes sociais, os humanos estão conectados numa grande rede virtual de relações sociais. A importância dessas redes se dá, sobretudo, devido ao potencial de circulação de agentes infecciosos, cuja transmissão pode ser rápida em grandes redes de relacionamento sexual, como ocorre no Chemsex, quando diante de práticas sexuais de risco, como o sexo desprotegido potencializado por inúmeras substâncias psicoativas, princi- palmente se ocorrer entre indivíduos soro discordantes ${ }^{(9)}$.

A categoria Principais substâncias psicoativas representou $32 \%$ dos achados, destaca-se que alguns estudos no Reino Unido demonstram que o sexo anal desprotegido entre gays e $\mathrm{HSH}$ é fortemente associado ao número de drogas consumidas durante um encontro sexual. Inclusive a probabilidade foi estimada de $25 \%$ de relações sexuais desprotegidas, quando nenhuma droga era consumida, e tal aumentava para $30 \%$ quando apenas uma substância era consumida, para $50 \%$ com o uso de substâncias e para $75 \%$ quando cinco ou mais drogas eram usadas ${ }^{(10)}$.

O Chemsex, é uma prática muito comum entre homossexuais, essa união ao consumo de entorpecentes é a provável causa do aumento dos casos de ISTs. Os adeptos descrevem efeitos de: euforia, aumento da energia, estado de alerta, urgência por falar, melhora da função mental, aumento da percepção da música, diminuição de sentimentos hostis e aumento do desejo sexual. O consumo de drogas como a metanfetamina ou a mefedrona provoca uma desinibição das emoções sexuais. Para além disto, drogas como o GBL (gama-butirolactona) matam uma pessoa a cada 12 dias na Inglaterra. Para entender a complexa relação entre sexo desprotegidos e drogas, é fundamental compreender a farmacodinâmica das drogas, bem como o contexto social $\mathrm{e}$ as perspectivas que o individuo tem diante da vida. Outras também podem ser consumidas como: cocaína, speed, ketamina, ecstasy ou MDMA. Quando as drogas são injetadas, o termo utilizado é 'slamsex ${ }^{\text {'(8) }}$.

A categoria Aspectos epidemiológicos, apesar do sexo desprotegido ser potencializado com o uso de substâncias entorpecentes, observa-se através da análise dos estudos que a Inglaterra é o país que mais apresenta problemas de saúde pública, sendo registrado em média 100 casos novos de HIV por mês de consumo problemático vincu- lado à prática do Chemsex. A Europa tem convivido com um surto de Hepatite A, o que tem preocupando autoridades que desconfiam da origem dessas moléstias em decorrência de festas que promovam o Chemsex. A gonorréia e a sífilis também são facilmente transmitidas através da prática sexual sem preservativo ${ }^{(8)}$.

Dentre os artigos pesquisados, verificou-se que de cada 387 homens que realizam 'Chemsex' ou 'Slamsex' e têm relações sexuais com homens HIV positivo sexualmente ativos, 50,6\% deles consumiu drogas de uso recreativo nos últimos 3 meses; $21 \%$ consumiram 5 ou mais simultaneamente; e $47 \%$ consumiram 3 ou mais simultaneamente. Em um estudo ${ }^{(9)}$ desenvolvido no Brasil, quanto aos comportamentos de risco para IST entre os homens, 10,3\% e $41,5 \%$ referiram ter fumado e ingerido bebida alcoólica nos últimos 30 dias, respectivamente; $15,0 \%$ experimentou drogas ilícitas alguma vez na vida, $19,1 \%$ não usou preservativos na última relação sexual e $66,1 \%$ afirmou ter tido dois ou mais parceiros sexuais.

$\mathrm{Na}$ categoria Infecções sexualmente transmissíveis/Morbidades, alguns dados já foram apresentados na discussão das categorias anteriormente descritas. De acordo com a OMS, mais de 30 bactérias, vírus e patógenos parasitas são transmitidos sexualmente. Epidemiologicamente, o contato sexual é mais importante para a sua transmissão de uma pessoa para a outra ${ }^{(11)}$.

De acordo com dados mais recentes, por ano ocorrem mais de $376 \mathrm{mi}$ lhões de novas infecções de clamídia, gonorréia, sífilis e tricomoníase. Esse número é praticamente o mesmo de 2012, o que mostra uma estagnação na redução da transmissão de ISTs. A cada dia são mais de 1 milhão de novos casos, tendo impacto profundo na saúde no mundo. Se não forem tratadas, podem levar a efeitos graves e crônicos à saúde, dentre os quais doenças neurológicas e cardiovasculares, infertilidade. Essas infecções também estão 


\section{artigo}

Silva, R.R.; Silva, L.A.; Silva, M.V.G.; Neves, M.P.; Silva, M.M.S.; Francisco, M.T.R.; Marta, C.B.;

associadas a níveis significativos de estigma e violência ${ }^{(11)}$.

Alarmante também foram os dados agrupados na categoria Abordagens terapêuticas, revelando que o baixo interesse e ausência de políticas públicas e disponibilização de recursos para o tratamento de pessoas que têm o comportamento de praticar o Chemsex. A dificuldade em encontrar locais para atendimento pode ser uma das justificativas para esse quadro ${ }^{(10)}$.

Faz-se importante conhecer a perspectiva cognitiva dos praticantes do Chemsex, seus contextos culturais para, então, realizar-se práticas que possam elucidar questões relacionadas à infecção consciente e reinfecção do vírus HIV e de outras ISTs e diminuir o preconceito em relação a estes indivíduos. Torna-se necessário conhecer o comportamento sexual e as condições de vida da população para analisar as necessidades e contribuir para a prevenção de novos casos de ISTs e a utilização consciente de preservativos durante esta modalidade sexual.

\section{CONCLUSÃO}

Através do presente estudo foi possível descrever o estado da arte das produções científicas sobre o Chemsex, bem como compreender os impactos desta modalidade sexual na saúde pública. A concepção do comportamento de risco e a banalização de métodos de barreira em detrimento da busca por prazeres sexuais prolongados têm colocado em risco a vida de muitas pessoas.

O Chemsex que, incialmente, era praticado entre gays e HSHs, atualmente pode ser verificado entre heterossexuais que promovem festas regadas a múltiplas drogas que possam potencializar e prolongar o desemprenho sexual e a realização de sexo com múltiplos parceiros e parceiras.

O Brasil carece de estudos sobre esta temática, almeja-se que este trabalho possa contribuir para o desenvolvimento de pesquisas de campo, as quais possam mapear a realização do Chemsex, a fim de que sejam implementadas medidas protetivas e preventivas para aquisição de ISTs.

\section{REFERÊNCIAS}

1. Alarcon S. A síndrome de Elêusis: considerações sobre as políticas públicas no campo de atenção ao usuário de álcool e outras drogas. In: Alarcon, S. (Org.). Álcool e outras drogas: diálogos sobre um mal-estar contemporâneo. Rio de Janeiro: Editora FioCruz; 2012. P 45-62.

2. Ministério da Saúde (BR). Boletim Epidemiológico - Hepatites Virais. Secretaria de Vigilância em Saúde. Departamento de Doenças de Condições Crônicas e Infecções Sexualmente Transmissiveis (DCCI). Brasília: Volume 50 | N² 26. Set. 2019

3. Dallo LM, Raul A. Associação entre as condutas de risco do uso de álcool e sexo desprotegido em adolescentes numa cidade do Sul do Brasil. Ciência \& Saúde Coletiva [Internet]. 2018 [acesso em 19 out 2019]; 23(1):303-314. DOl: https:// doi.org/10.1590/1413-81232018231.14282015.

4. Barreto VHS. Limites, fissuras, prazer e risco em festas de orgia para homens. Mana [Internet]. 2019 [acesso em 20 out 2019]; 25(1):9-37. Disponivel em: http:// www.scielo.br/scielo.php?script =sci_arttext \&pid=S0104-93132019000100009\&lng=en\&nrm=iso.

5. Silva NEK, Freitas HAG, Sancho LG. Da apreensão de informações aos itinerários terapêuticos de homens diante de suspeita ou com diagnóstico de infecções sexualmente transmissiveis. A internet em pauta. Physis [Internet]. 2016 June [cited 2019 Oct 20]; 26(2):669-689. Available from: http://www.scielo.br/scielo.php?script=sci_arttext\&pid=S0103-73312016000200669\&Ing=en.

6. Galvão TF, Pansani TSA, Harrad D. Principais itens para relatar Revisões sistemáticas e Meta-análises: A recomendação PRISMA. Epidemiol. Serv. Saúde [Internet]. 2015 June [cited 2019 Oct 20]; 24(2):335-342. Available from: http://www.scielo.br/scielo.php?script=sci_arttext\&pi-

\section{d=S2237-96222015000200335\&Ing=en.}

7. Rios LF, AlbuquerqueAP., Santana W, Pereira AF, Oliveira Junior CJ. (2019). O drama do sexo desprotegido: estilizações corporais e emoções na gestão de risco para HIV entre homens que fazem sexo com homens. Sexualidad, Salud y Sociedad [Internet]. 2019 Sep [acesso em 09 set 2019]; 32:65-89. DOI: https://dx.doi.org/10.1590/1984-6487.sess.2019.32.05.a.

8. Chavez KAP, O'Brien B, Pillon SC. Uso de drogas e comportamentos de risco no contexto de uma comunidade universitária. Rev. Latino-Am. Enfermagem [Internet]. 2005 Dec [cited 2019 Oct 20]; 13(spe2):1194-1200. Available from: http://www.scielo.br/scielo.php?script=sci_arttext\&pid=S0104-11692005000800014\&Ing=en. http://dx.doi. org/10.1590/S0104-11692005000800014.

9. Neves RG, al. Simultaneidade de comportamentos de risco para infecções sexualmente transmissivveis em adolescentes brasileiros, 2012. Epidemiologia e Serviços de Saúde [Internet]. 2017 [acesso em 20 out 2019]; 26(3):443-454. DOI: https://doi.org/10.5123/S1679-49742017000300003.

10. Silva NEK, Freitas HAGS, Leyla G. Da apreensão de informações aos itinerários terapêuticos de homens diante de suspeita ou com diagnóstico de infecções sexualmente transmissiveis. A internet em pauta. Physis: Revista de Saúde Coletiva [Internet]. 2016 [acesso em 20 out 2019]; 26(2):669-689. DOI: https://doi.org/10.1590/S0103-73312016000200016.

11. Organização Mundial da Saúde. Diagnóstico laboratorial de doenças sexualmente transmissíveis, incluindo o vírus da imunodeficiência humana. Coordenação de Laboratório do Departamento de DST, AIDS e Hepatites Virais, 2014. 